\title{
Application Method of Struts Framework Based on MVC Design Pattern
}

\author{
Kangle Zhou \\ Nanchang Institute of Technology, 330044, China
}

Keywords: MVC Mode, Struts Framework, Application Research.

\begin{abstract}
A good design pattern can effectively build the system structure and reduce the complexity of the system. Model view controller (MVC) is a framework level design pattern that can separate data access and data performance. This paper introduces the development, concept and implementation of MVC pattern, and describes the specific application of MVC pattern in Struts framework in detail.
\end{abstract}

\section{Introduction}

With the development of society, the development of web-based application system has developed from the past C / S mode to the present B / S mode. Web based applications include online transactions, information management, workflow and common environment, online communities and more[1]. They have the common functions of multiple web pages, complex browsing and complex business processing logic. The development of web application system based on B / S model is very important for using highly reusable and easy to extend and maintain components. Therefore, the struts framework under MVC model is used to divide complex and huge problems into smaller processing modules. This paper introduces the basic concept and architecture of Struts framework, and analyzes the advantages of the system developed by the framework.

\section{MVC Mode Overview}

MVC pattern is a software design pattern put forward in 1980s. MVC consists of three parts: model, view and controller. There are business logic and business rules in the model. For example, JavaBeans, EJB, and other component technologies can be used for database transactions. The model can also provide data for multiple views[2]. The code for the model can be reused in multiple views by writing only once. Then that improves programming efficiency. Views are used for output and display. After using the operation model, the results can be output and displayed through the view, but the actual processing operation will not occur in the view. Controllers are used to manage the interaction between users and views. If the user wants to work with the model, it can be performed by the controller. The controller can get the value of the view and send the corresponding value to the model for processing. The controller can accept user input, then call the model and view to complete the user. Demand. Three components of MVC mode, we can understand the operation process of introducing MVC: first, controller sending requirements, controller is the mode selection of operation, and in order to respond to the mode, user requirements, the processing result is to repay the use of business logic[3]. Finally, the corresponding view is used to output the user's browsing results. MVC itself is very complex.

\subsection{Struts Framework}

MVC reuses Java developers to develop web applications using J2ee. Like other Java based frameworks, Struts framework is a software development and object-oriented design method. The struts framework combined with MVC makes full use of the characteristics of MVC[4]. Struts framework is independent of controller, model and garden layer encapsulation for other use technology. The struts framework provides many places for extension and customization, as well as a customized tag library. 
MVC can be used as a mainstream framework in many web application systems. The main reason is that it has many advantages. MVC can build a running model and use multiple views at the same time. MVC also has a special mechanism, called internal data monitoring mechanism, which depends on the object pattern. Update and update the data in the view layer for synchronous response.

MVC has many advantages, but also some disadvantages. For example, it makes the system structure more complex and increases the difficulty of implementation. In addition, MVC is suitable for large-scale systems. If the functional structure of the system is relatively simple, if the MVC mode is strictly followed, the complexity of system configuration will be increased[5]. Also, it can be caused by more updates. As a result, the efficiency of the system is reduced. In addition, the coupling between the view and the controller is too strong. In theory, view and controller are separated from each other, but in practical application, the coupling between view and controller is relatively strong. Without the controller in the view, his applications would be very limited, and vice versa. This strong coupling prevents the independent reuse of components. In addition, the efficiency of accessing views of data through model interfaces is very low. This is because the interfaces in the model layer are different. Therefore, in order to obtain sufficient display data, multiple displays are usually performed. The data efficiency of the access method is relatively low, and it also has a certain impact on the operating performance of the system. In summary, through the analysis of MVC mode, we find that the role of MVC mode is very obvious, it is more obvious in the large system structure.

\subsection{MVC Mode Basic Implementation Method}

There are one or more ways to implement the MVC pattern. For example, a framework that uses MVC patterns such as struts and JSF is an implementation in itself. This paper mainly introduces the simple basic implementation method.

\subsection{Implementation of Model}

In the implementation of the model, the most important thing is to define the vector of the audience object that stores multiple data updatesand then according to this vector, plus the score of audience and object in data service, in order to implement the following two functions, when the update cannot be written, all information can be pulled.

\subsection{Implementation of View}

There are three key points in the implementation of view. First, when executing view consur, you need to register the view with the specified model object[6]. The second point is to define controller objects that can store controller vectors. In addition, it is necessary to define the login method of the additional action audience according to the object of this vector. Finally, you need to show different types of views that can show different types of interfaces. Through the above three key points, the view can receive model update notification, send operation to the controller, and accept the controller's control over the display interface.

\section{Three Struts Frameworks Based on MVC Pattern}

The so-called struts framework based on MVC pattern integrates javavlet, JSP, taglib, JavaBeans, XML and resource balls into a framework to form a reusable MVC design. The struts framework is equipped with a unique controller and integrates other technologies to implement model layer and view layer. Moreover, the struts framework provides a lot of places for extension and customization. At the same time, the struts custom tag library is provided[7]. These custom tags can interact with the model part of the system. With these custom tags, you can use ActionForm to implement the mapping in the model section. Encapsulation of user data.

Struts framework controller (actionservlet). When the main controller starts, first read the setting information of struts config. XML, and initialize different struts modules of corresponding objects. If there is a corresponding action and the action has a corresponding ActionForm, the ActionForm 
is instantiated by the controller first, and its properties are satisfied by the data requested by HTTP, and then stored in the ServletContext. The controller switches the request information to the specific actionforeman according to the setting information and passes it to the execute() method of the action[8]. The action is usually responsible for executing the corresponding business logic, including the execute() method that returns the actionforward object, which is used by the controller for operation. Different results are generated between business processes. According to the action, the object corresponding to the overall control goal is returned to you. Then, the object corresponding to the goal is a specific JSP page or other action response. The general controller converts the HTTP request to the target response object according to the target response object returned by the business function action. Generally speaking, it is a specific JSP page. The target object response object (JSP) prompts the user for a result page.

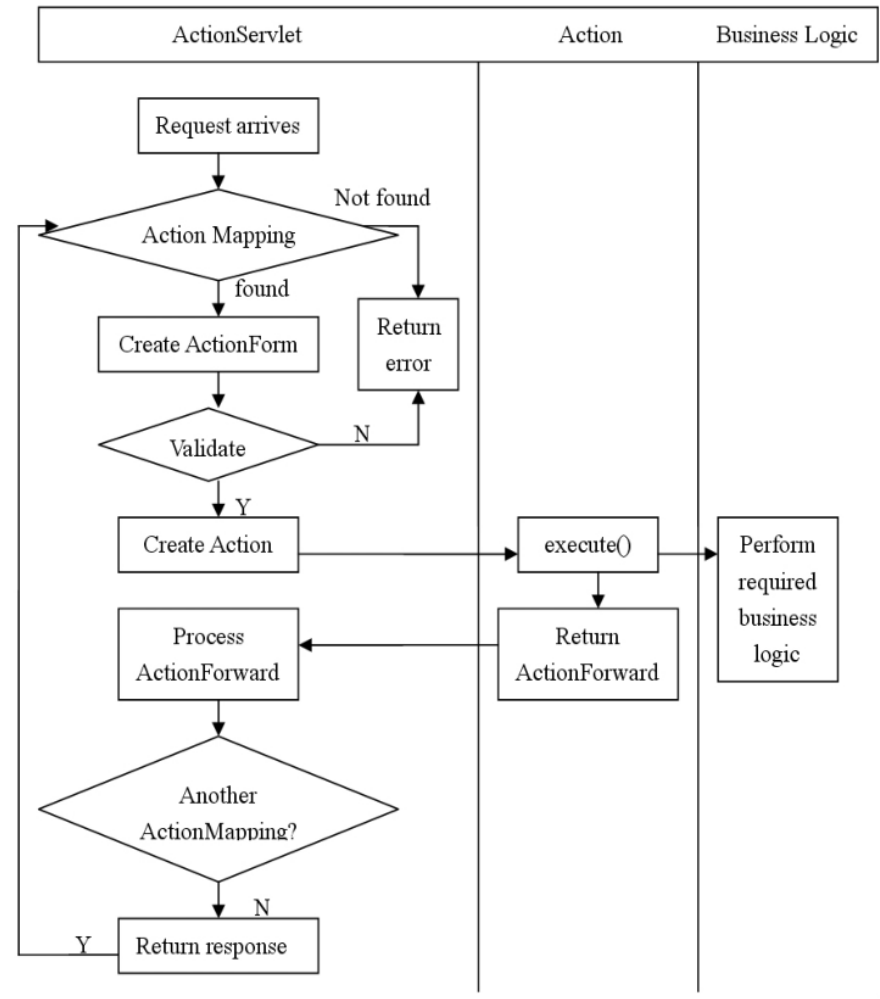

Figure 1 Workflow of struts in response to user requests

\subsection{Web System Architecture Design}

The design concept of MVC mode and struts framework reveals that the online forum, hierarchical design, is employed to make the layer, control layer, business logic layer, data access layer, and garden layer, that is to say, the interface referring to user interaction is designed as interaction. The view layer accepts the HTTP request submitted by the user and sends it to the control layer for processing. In general, the Action form bean of the struts framework is used to implement this data transfer. The function separates the required control layer from the business logic, and is responsible for controlling the application process and calling the method of the business logic layer. In general, install the actionservlet and action classes of the struts framework. The business logic layer is the core of this web system. According to the control flow of the control layer, the data provided by the data access layer is used to execute business rules[9]. Business models are generally built by JavaBeans, including system state control and business logic control. According to the requirements of the business logic layer, the data access layer provides transparent data access to the upper layer. The program unit based on object access and the table access the fault match based on data unit. In order to solve the problem of additional data of ORM (relational mapping) mechanism in data access layer, the lower unit implements the unified processing. In general, to achieve the above purpose, Java is used for the state bean class and Dao class corresponding to each database table. From the above analysis, referring to the MVC model and 
struts framework, the hierarchical Online Forum web system is designed. The combination between the layer and the high flocculation in the layer is low, flexible, and it is possible to reuse, and it is easy to expand the possible web system construction. Moreover, it improves the efficiency of web application system development, reduces the complexity and is easy to realize.

\subsection{Controller Implementation}

Compared with the implementation of model and view, the implementation of controller is very simple. If it's a controller, you only need to define model objects and view objects. Model, object is its function of business behavior call, action receiver view, controller action, in order to make registration easy.

\section{Conclusion}

In summary, MVC model has been widely used in software development. This can be visualized by making the program structure more complex and simple. The software system also separates the basic parts of the software system, and the basic parts will give them corresponding functions. Using struts framework based on MVC pattern can greatly improve the flexibility of control layer and the reusability of code. The complex system based on the struts framework of MVC pattern can separate data access and data performance. Developers can then develop a scalable, simple extension controller to maintain the entire process. Therefore, in the complex structural system, Struts framework based on MVC pattern has great advantages and development prospects.

\section{References}

[1] ZHAO, Lin., WANG, Hongxia. Research and Application of the Web System Based on Spring MVC+JDBCTemplate. Software Engineering, 2017.

[2] Juho-Ville, Kinnunen., Harri, Piitulainen., Jarmo, M, Piirainen. Neuromuscular Adaptations to Short-Term High-Intensity Interval Training in Female Ice-Hockey Players. Journal of Strength \& Conditioning Research, vol. 33, no. 2, pp. 1, 2017.

[3] Yuta, Kato., Atsushi, Iwata., Masayuki, Nakamura. In-Stent Restenosis due to Stent Recoil After Third-Generation Drug-Eluting Stent Implantation. Journal of Clinical Medicine Research, vol. 9, no. 6, pp. 534-538, 2017.

[4] Liu, H.P., Duan, S.M. MVCR Performance Improvement Research Based on ANSYS, 2018.

[5] Yang, S., Wei, J., Zhao, X. Study and implementation of scientific research project assessment system on network based on text mining, 2017.

[6] Yang, S., Wei, J., Zhao, X. Study and implementation of scientific research project assessment system on network based on text mining, 2017.

[7] Hyun-Jun, Jang., Jung, Dae, Lee., Hyun-Sik, Jeon,. Metabolic Profiling of Eccentric ExerciseInduced Muscle Damage in Human Urine. Toxicological Research, 34, 2018.

[8] Black, Christopher, D., Schubert, Daniel, J., Szczyglowski, Marcin, K,. Carbohydrate Mouth Rinsing Does Not Prevent the Decline in Maximal Strength After Fatiguing Exercise. Journal of Strength \& Conditioning Research, no. 32, 2018.

[9] Boris, Ullrich., Thiemo, Pelzer., Mark, Pfeiffer. Neuromuscular Effects to 6 Weeks of Loaded Countermovement Jumping With Traditional and Daily Undulating Periodization. Journal of Strength \& Conditioning Research, vol. 32, no. 3, pp. 1, 2017. 